\title{
The Representation of Time in Agency
}

Holly Andersen

Simon Fraser University

holly_andersen@sfu.ca

\begin{abstract}
This paper outlines some key issues that arise when agency and temporality are considered jointly, from the perspective of psychology, cognitive neuroscience, phenomenology, and action theory. I address the difference between time simpliciter and time as represented as it figures in phenomena like intentional binding, goal-oriented action plans, emulation systems, and 'temporal agency'. An examination of Husserl's account of time consciousness highlights difficulties in generalizing his account to include a substantive notion of agency, a weakness inherited by explanatory projects like neurophenomenology. I conclude by sketching a project analogous to the projects in neurophenomenology, based on Thompson's naïve action theory.
\end{abstract}

Keywords: time; agency; representation; temporal; action; explanation; Husserl; Thompson

\section{Introduction}

Agency is fundamentally temporally structured. We form intentions to do something, resolutions that are held now to bring about something in the future. We meet with the right conditions to implement those intentions and we act. Our actions are executed through time, in temporally extended processes requiring attention until the actions are complete. Our doings as agents in the world are irreducibly temporally extended, involving both time itself as well as various representations of temporality.

There are three distinct elements this paper will disentangle in order to draw out the connections between them: temporal experience, agency, and representation. The phrase 'temporal experience' covers a range of phenomena. For the purposes of this paper, we should note that it includes: the temporal character of all experience, namely, that whatever we experience, we experience as 'now'; the perception of time, when we attend to or are otherwise aware of the passage of time itself; and the explicit representation of various features of time in experience, such as duration, frequency of events, sequence of events, etc. Since agency as implemented in our lives is fundamentally temporally structured, it is useful to see how the temporal 
structure of agency fits into the broader temporal structure of experience. And both in experience more broadly and in agency specifically, time can play distinct roles based on whether it is explicitly represented. ${ }^{i}$

The goal here is not to provide an overarching account of the role that the representation of time plays in human agency, nor a complete survey of the incredibly varied and numerous ways in which temporality is involved in agency. Rather, I will outline some of the key issues that arise when agency and temporality are considered jointly, and will trace out some intriguing paths for future work from the tangle of issues involved in the representation of time in agency. Although accounts of agency and temporal experience have often been developed independently, considering them together enables us to use one to shed light on the other, and highlights lacunae in our understanding of the character of temporal experience and its neurophysiological underpinnings. Once we get a sense of the ways in which these two topics mutually inform and constrain one another, we will consider the ways in which the representation of time in agency adds complexity over and above the mere involvement of time in agency: we can then ask, what is the role of represented time in agency, rather than time simpliciter.

While they are not exhaustive, and in some cases not exclusive, there are three primary levels at which distinct clusters of issues can be identified for the relationships between time, agency, and representation. One is psychological: questions here may concern how the representation of the temporal sequence of events changes when agency is involved, comprising both personal and subpersonal mechanisms. The second is phenomenological: what is the precise content of our temporally structured experiences as agents? The third level is neurophysiological: can we ground the character of phenomenological and pyschological accounts of time and agency in various neurophysiological processes? ${ }^{\mathrm{ii}}$

\section{Connections between time, agency, and representation}

The issue of representation, temporal or otherwise, is closely tied to the issue of agency, in that representation is often taken to be a necessary condition for a creature to be capable of genuine agency rather than mere behavior. And what is required for genuine representation is itself an area of disagreement, particularly on the issue of whether rationality is a requirement for genuine representation, genuine agency, both, or neither. Some philosophers hold that only full-fledged rational creatures, with adequate conceptual resources and reasoning abilities, can be meaningfully said to engage in representation (e.g. Brandom 1994, especially chapter 4). These philosophers are also committed to the view that there is a sharp distinction between mere behavior and agency, properly speaking, such that only rational, conceptually endowed creatures can be agents. That of which rational human agents are capable constitutes a fundamentally different kind of activity than the cognitive and behavioral activities in which animals, even higher primates, 
engage. Once a creature can conceptually represent the world and recognize inferential relationships between beliefs, it opens up fundamentally new ways of engaging with that world.

On the other hand, some philosophers (see especially Burge 2010) argue for continuums of both representational and agency capabilities, stretching from humans at the most developed end, towards other primates with markedly reduced capacities, and on towards mammals, birds, reptiles, and other species with everdiminishing capacities for either agency or full-fledged representation. There is a sharp divide between representing and non-representing creatures, but this divide occurs far down the phylogenetic tree. On this sort of view, humans are capable of the most refined forms of representation and agency, but there is no radical break our capacities and those of many other animals.

The question of whether representational capacities require rational or conceptual resources is in many ways prior to the question of whether a given creature can display agency or merely engage in behavior. Most accounts of agency involve representational resources in some fashion or another, such as the representation of an intended outcome or of means to achieve an end. Thus, if a creature is deemed incapable of genuine representation, it is precluded from exercising genuine agency. It may be that mere egocentric representation of the environment is insufficient for agency: perhaps a creature also needs to be able to represent itself as an element in an objectively represented environment and as having specific capacities to move about and act in that environment. If one subscribed to such a view of agency, representation would be necessary but insufficient for agency.

We can bracket this issue by noting that when we move from creatures that merely involve time in perception or behavior to those that explicitly represent time, there is a corresponding increase in a host of other abilities with respect to representation and agency. Since there is agreement that we are capable both of agency and of genuine representation, and since this is the focus here (note the lack of examples from orangutan research), we can safely turn to consider the ways in which time plays an explicit role in human agency.

There are some obvious ways in which temporal representation is involved in agency. One is that intentions are directed forward in time at some action. Forming an intention involves representing as in the future some action that one plans to execute. The forward-directed nature of agency can either bring about or may presuppose a certain degree of diachronic rational consistency, since an agent that forms an intention must sustain that intention through time and implement it at some time after the intention was formed. Changing our prior intentions without any change in our reasons for having formed those intentions is a species of irrationality, distinguishable from, for instance, simultaneously holding contradictory beliefs. Agency provides temporal structure and continuity through time, which has been offered as the basis for the constitution of a rational, unified, 
self, one which persists through time as the same self (see, inter alia, Korsgaard 2009, Hinchman 2003, Zahavi forthcoming).

As an example, consider Bratman (2007). "We are reflective about our motivation. We form prior plans and policies that organize our activity over time. And we see ourselves as agents who persist over time and who begin, develop, and then complete temporally extended activities and projects" (Bratman 2007: 21). In order for something like an attitude towards a desire or goal to have "agential authority", or, to carry normative force for how the agent with that attitude ought to act, such an attitude must participate in "complex continuities and connections that help constitute the organized interweave of our action and practical thinking over time" (2007: 5). An agent must recognize that she is herself temporally persisting in order to engage in a number of activities that are integral to agency. One acts now in order to bring about some good in the future because one sees oneself as the recipient of that good. One organizes one's actions under long-term plans and policies that structure motivations and shorter-term intentions. Note that while these are temporal activities, it not merely time that is involved in these constitutive features of agency. It is temporal representation as a framework for structuring current actions (this is what a policy is, for instance), as well as an explicit temporal element in representations of the self, other agents, of elements of the environment in which one acts, and so forth.

These are just a small handful of ways in which temporal representation plays a role in agency, personal identity, and rationality, in traditional philosophical approaches. Rather than attempt to cover the myriad ways in which temporal representation is involved in these discussions, I will instead consider some intriguing results from the sciences that bear on the question of temporal representation and agency.

\section{Time, agency, and representation in the cognitive sciences}

There are tight connections between action and perception that shape the way in which we perceive temporal relationships and the way in which time passes. Though this connection certainly runs from perception to action - we first perceive various features of the world and then act based on that perceptual information the connection also runs the other direction, where action shapes perception in a variety of ways, including the perception of time.

One straightforward example of how action shapes perception is that of intentional binding: we perceive events as occurring closer in time than they actually did when those events are an action of our own and its effect. When subjects estimate the time elapsed between events they did not bring about, they are more accurate than when they estimate the time elapsed between their own movements and the effects of those movements. When the two events to be timed are the actions of the subject and the consequences of those actions, there is a systematic bias towards shortening 
the time between them - subjects experience the two events as bound closer in time than they were (Haggard and Cole 2006).

Intentional binding indicates that temporal perception is responsive to agency: when we act, we perceive the effects of those actions as closer in time to our actions than we do other events that were not under our control. Recently, this effect has been offered as an indirect measure of the sense of agency (Ebert and Wegner 2010). The sense of agency, sometimes called the feeling of authorship, is the subjective experience had when an agent perceives her action as the cause of the subsequent event. Manipulating parameters such as temporal contiguity of action and effect changes how often subjects report experiencing the sense of agency with respect to those effects. While this measure does not provide information on the mechanisms by which action influences temporal perception (Moore, Wegner, and Haggard 2009; Moore and Haggard 2010), the fact that some mechanism(s) does result in binding may still be useful in predicting the degree to which subjects feel authorship over their own actions.

This highlights the fact that the sense of agency does not simply track the movements that we in fact generate in order to bring about intended effects. It at least partially tracks the temporal evolution of effects for which we are potentially responsible, and assigns authorship based on temporal parameters like contiguity in time between movement and effect. There can be a surprisingly wide margin of difference between how we actually move our arm and how it misleadingly appears to us, through carefully manipulated visual feedback, that we move our arm, before we notice the discrepancy (Preston and Newport 2010). The sense of agency can be induced when the intended goal of a movement is achieved, even if it was not the subjects' actual movements that achieved it, so long as it occurred in close temporal proximity to the movement.

There is substantial evidence to the effect that many of the processes that ground action (including, most basically, our abilities to move our bodies) are generated in ways that involve a splitting of motor signals in the brain, such that one signal serves as the control for muscle movement, while the other signal is a copy that generates the feeling of moving those muscles, or an awareness that they are about to move (see David, Newen, and Vogeley 2008; Kandel, Schwartz, and Jessel 2000: chapter 33). The sense of agency arises when perceptual feedback reveals coherence between the expected outcome, based on predictions about the motor activity derived from a copy of the efferent signal sent to the muscles, and the actual outcome, as ascertained from perceptual information about limb position and movement and kinesthetic feedback. Thus, anything that caused the actual outcome to diverge from the prediction would reduce or eliminate the sense of agency associated with that outcome, whether or not one did in fact cause the actual outcome.

This dissociation of the sense of agency from self-generated movements is to be expected when considered in light of other results. Evidence from psychology and 
cognitive neuroscience suggests that intentional actions are implemented as part of an ongoing, more abstract, action plan (see, e.g., Hommel 2003; Kandel, Schwartz, and Jessel 2000). Action plans are formulated in terms of an intended goal or outcome state that the action will achieve. These plans may be general in character, such as grasping an object on the table. Unconscious systems for sensory-motor processing implement this general plan in precise movements of the hand, in this case by signaling to hand muscles to form the right shape and arm muscles to reach in the correct direction. Thus, an agent does not micromanage the details of how precisely she moves her hand in order to grasp the object; she knows that she is moving her hand, and that the object has been successfully grasped. Since the action plan represents the action in terms of the outcome state, one would predict subjects feeling the sense of agency or authorship over a successful object-grasping action, even when they may not have accurately perceived their own arm movements in doing so.

Skillful execution of complex, fast-paced sequential movements - e.g., dancing, piano playing, or racquetball - rely on a number of factors such as precise action plans and the kind of automaticity that results from deliberate practice. It is impressive that our brains can manage in real time the amount of perceptual and motor information necessary to engage in these complex actions. The emulation theory of representation (Grush 2004) gives us a handle on these kinds of tasks in terms of information processing, independently of the mechanisms by which it is implemented. On the emulation theory, sensorimotor information is processed in a feed-forward/feedback representational structure with both motor and sensory loops. Information about how the environment and body respond under various conditions is encoded in the representational structure as a mathematical function, from which predictions can be derived for outcomes given various efferent signals in a given environment. The predictions made by an emulator that had only been exposed to the gravitational field on Earth would differ from the predictions made by an emulator that had only been exposed to the gravitational field on the moon, for instance. On the moon, a dropped pen would fall more slowly, and thus an appropriate reach-and-grasp motion would be aimed at a different location in the downward trajectory; similarly, a well-placed kick would have to be initiated differently, and be guided through to completion differently. Emulator representation systems incorporate sensory information and copies of efferent signals sent to muscles in order to plan actions that achieve certain goals, where goals might be states of the environment - a returned serve - or a particular position of the body.

Emulator information processing systems not only involve time but rely on representations of time. They are temporally structured in that emulation is a multistep process that necessarily takes place over some period of time, with input stages that have to be separated by temporal intervals in order to successfully function in guiding skilled physical action through to completion. And they involve temporal representation in that time is a key variable in most, if not all, equations representing information about how the body and environment respond. 
Furthermore, if the emulation theory of representation captures genuine features of information processing, then we have further evidence that the experience of agency will vary separately from self-generated bodily movements, since each depends on a different set of factors. In the emulation theory, if a subject were to move her arm and hand in a reach-and-grasp motion, but be given visual feedback that indicated their arm taking a different course than it actually did (see the experiments mentioned above), the emulator system would be given conflicting information about the actual movement, and the apparent movement would not match the predicted movement based on the copy of the efferent signal. In other words, the system would not process that apparent movement as something it 'did'.

These examples illustrate several general points about time and agency. They highlight the temporal extension of human action, and the irreducibility of action to single instants. Actions, even short bodily movements, are not temporally punctate events. Rather, they involve a fairly complex chain of events that includes motor planning, with conscious and unconscious stages, drawing on perceptual information that may have been gathered specifically for the purposes of planning a particular movement, and which continues to guide that movement through to completion of the effect goal. Furthermore, those action plans shape perception in several ways throughout the process of development, implementation, and guidance through completion (Fagioli and Hommel 2005).

The dissociation between agency and the sense of agency also has philosophical implications for investigations that attempt to ground conscious agency in neurophysiological processes. Most importantly, it entails that the sense of agency is not an adequate proxy subject if one intends to study agency itself. While it's not clear where we should outline the division between genuine agency and 'everything else', in cognitive neuroscientific terms, it is clear that agency as it has consistently been discussed does not map solely onto the sense of agency alone, but would include at least some of the premotor and motor processing that results in limb movement, for instance. This implication of the dissociation between agency and the sense of agency will be explored in greater detail in subsequent sections.

Considerations of time in terms of action plans, intentional binding, or emulator models, however, involve a fairly weak notion of representation. While information processing accounts like the emulation theory of representation may indicate an important way in which temporal representation is involved in agency, there is nothing explicit about this representation of time - it may not ever be part of the content of conscious experience, but merely part of a subpersonal mechanism. For instance, we may not even be capable of becoming aware of the fact that we've experienced two events as closer in time than they actually were; we may not be able to reconstruct how we were able to successfully catch something so quickly and accurately, even though we did it 'on purpose'. The next step is to consider the ways in which explicit representations of time figure in agency. 
One way in which representations of time can figure in agency is by themselves serving as the object to which we apply ourselves as agents, in order to bring about changes in how time is represented. An intriguing connection between temporal experience and agency emerges in the context of the ways in which humans actively arrange their activities, including thinking, in order to change the phenomenal character of their own experience of time. Michael Flaherty calls this "temporal agency" (2011), namely, directing one's agency at one's own experience of time in order to change that experience. We are capable of exerting some degree of control over a number of distinct parameters along which temporal experience may vary, in order to bring about changes in that experience.

Flaherty provides details from interviews the tactics and tricks people use to manage their daily lives, and finds that many of these fall into broad categories based on how they change our perception of time and experience of events through time. For instance, everyone is familiar with the phenomenon of the interminable lecture that goes on and on, where the passing of each minute seems to take forever. One may become acutely and uncomfortably aware of the passage of each moment; one may decide to change the character of that temporal experience in order to speed up the apparent duration of the lecture. Students report a variety of tactics for "passing the time," an activity in which they deliberately engage for the purpose of making the lecture seem to pass less slowly. Conversely, agents sometimes have periods of time that appear to pass very quickly, and engage in tactics to prolong an enjoyable period of time that may otherwise end too soon.

Other characteristics of our experience of time on which we can exert some control as agents include the frequency with which certain events occur, their sequence or order, and their timing. Acting on each of these parameters involves explicitly considering the way in which one's experience of time is structured, as well as considering the temporal relationships of events in time. Time is also something that agents can allocate: subjects reported various strategies for choosing how to spend different periods of time in order to make room for studying, fun activities, or family time. Of course, no one has complete control over any parameter of how time passes. It is useful to consider, though, how many of our daily activities that often are not registered as important acts of agency are nevertheless deliberate and can be understood as involving a limited alteration of some facet of one's own experience of time.

\section{Agency and the phenomenology of temporal experience}

No discussion of the representation of time would be complete without considering the phenomenological account of time consciousness developed by Edmund Husserl. By considering Husserl's work on temporal consciousness in light of the question of agency, we can highlight how the analysis that he provides, while well- 
suited for the purposes to which he puts it, cannot be extended to include agency in its ambit.

Husserl's On the Phenomenology of the Consciousness of Internal Time is easily the most influential single work on the character of temporal experience in this tradition. While there isn't space here for a full investigation of Husserl's account, there are several features that are especially germane to the issue of temporal representation and agency. He lays out the fundamental features of what he calls the absolute, time-constituting flow of consciousness in terms of a tri-partite structure. The structure of the 'now' in experience has three distinguishable phases,: the leading edge of protention, the central primal now, and the fading-out of retention. The present moment, as experienced, thus spans some nonzero length of time, both in absolute terms - if one timed it with a stopwatch, it would be nonzero - and in phenomenological terms - it is experienced as stretching over some duration.

The primal now is the most central and immediate part of the now. It is the part we would be most inclined to associate with the instantaneous 'now' as timed with a stopwatch. Protention is the forward-looking element of the now, directed at incoming experience. Protention should not be understood as a literal experience of the future, but it is future-directed and involves sometimes substantial structuring expectations about imminent experience. The receptivity to imminent experience, and the expectations regarding what is just about to happen, are protentive in character. Retention is less controversial than protention, as the lagging edge of experience that fades into the past. The way in which immediately present experiences fade into the past, rather than disappear instantaneously, illustrates retention.

A key feature of the absolute time-constituting flow is its double intentionality. The 'now' is always intentional in character, in a sense Husserl took up from Brentano (1995): it is about something, whether directed at something in the world or purely internal to the experience itself. Double intentionality means that the flow of the now, which is itself about something, can also be directed at itself, at other moments besides the present one. One moment of experience may take as its intentional content a different moment, which had its own intentional content. Remembering something involves double intentionality: this present moment intends a different section of the flow, that of the event being remembered. Double intentionality involves the representation, in the threefold structure of one present moment, of a different moment that itself had such a threefold structure. Thus, the representation of time has a double aspect in Husserl. The present both has a threefold structure, and represents itself as having a threefold structure.

It is a striking feature of Husserl's account of time consciousness that so many agency-oriented questions have no clear answer in the original text. iii One element of Husserl's account that might be thought to address this issue is his treatment of acts of knowing or acts of perception as temporally extended processes, and as constituted out of the absolute time-constituting flow of consciousness. He contrasts 
the way in which the content of acts of knowing are given to the subject of experience with the way in which objects thereby known are given (Zahavi 2003:13). Acts of perception or knowledge have objects that are given to the subject perspectivally - any given presentation of an object will not exhaust that object, since the object will have a hidden or obscured side that is not given in this presentation (even though that hidden side may be given in a different presentation). In contrast, acts are given nonperspectivally: they do not have a hidden side, but are given in their entirety in the act itself. Acts thus construed do not "reach out" to the world or transcend the experiences in which they are given they are immanent in experience. This is a very different notion of 'act' than has been considered in this paper. While it might be tempting to consider Husserl's treatment of 'acts' as implying some kind of agency, the term is agent-neutral, more akin to acts in a play than to actions.

There are methodological reasons why questions about agency may lie beyond the project Husserl set out. He sets up his investigation of time consciousness by bracketing the external world. This bracketing specifically sets aside questions related to the veridicality of experiences (such as, does the present moment 'really' have a nonzero duration?), and of the genesis or causes of such experiences (such as, what in our brains makes it the case that the present moment has a threefold character? or, what in the universe are we perceiving when we perceive the present moment?). Phenomenological bracketing severs the causal tie between subject and world on the incoming side - from world to subject. But in doing so, Husserl's investigation also precludes investigation of causal ties on the outgoing side - the way in which the phenomenological subject might intervene on or participate in the world as an agent.

These observations are not criticisms per se, but rather reminders of the inherent limitations of Husserl's phenomenological project. To a large extent these limitations are inherited in recent attempts at developing a project of neurophenomenology, an attempt to ground various elements of the tripartite account of time consciousness in specific neurophysiological processes (Lloyd 2002, Varela 1996, Lutz and Thompson 2003, van Gelder 1999; Gallagher and Zahavi 2008). iv

In neurophenomenology (Varela 1996), the goal is to use the tripartite analysis as an explanandum in order to isolate those representational vehicles in the brain that are responsible for these various aspects of temporal experience. Neurophenomenologists start by taking Husserl's account of time consciousness as a careful and basically accurate description of the experience of time, isolating key elements such as protention or retention, and then turn to neuroscience in order to seek out the specific processes in the brain or nervous system that might give rise to these characteristics of temporal experience, using EEG, fMRI, PET, and related measurement tools. 
As we just saw, Husserl's account does not presume the empirical adequacy of temporal experience. However, since questions of veridicality and causation are bracketed, the phenomenological method delineates precisely what should be explained in a neuroscientific account. Whether or not our experience is veridical, it is the phenomenological structure of temporal experience itself that now needs to be accounted for. This means looking for the physiological processes that ground or give rise to the temporal content of particular experiences, using the constraint that such information is at one moment presented as primordially now and at some later moment presented as having just happened. The right representational vehicle in the brain should be able to account for the content in current experience at any given moment, as well as the characteristics of how that content is represented - as right now, as just past, or as looming in the immediate future. Even though the representational vehicle(s) will involve further temporal structure than does the experience so represented, all the temporal features of the experience should figure in a neuroscientific explanation of that experience.

There are constitutive, evidentiary, and explanatory connections between carefully described aspects of experience and neurophysiological processes that can be traced out. These projects must walk a fine line with respect to the distinction between the content and the vehicle of a representation while still relying on features of the content to guide in the work of isolating the corresponding features of the vehicle (see Dennett and Kinsbourne 1992). The vehicle of a representation might have properties that do not reflect features of that which it represents; as a result, we should resist inferring from the fact that a representational vehicle has certain properties to the conclusion that these properties are reflected in the representation content. For a critical assessment of neurophenomenology in this regard, see (Grush 2006).

The agency-related questions that either did not arise or had no clear answer in Husserl's account are thus inherited by neurophenomenology. Insofar as this approach accepts the phenomenological bracketing as a means of delimiting the explanadum for cognitive science, it also falls prey to the flaw of dividing the subject of experience from potential causal interaction with the world. If one is solely concerned with temporal consciousness, this may not be a shortcoming. If, however, there is to be any prospect of generalizing this approach to explain further features of experience, pertinently questions relating to temporal representation involved in agency, we must move beyond the Husserlian approach. Even though interesting results have come from bringing Husserl's tripartite structure of time consciousness into contact with neurophysiological investigations, this approach as it stands lacks the ability to incorporate full-blooded agency into its analysis.

As an illustration, consider how one might think that the neurophenomenology project could be expanded by adding the phenomenology of agency to the phenomenology of time consciousness (see, e.g., Gallagher and Zahavi 2008). Even though this is a prima facie appealing solution, as discussed in the previous section, the phenomenology of agency is a non-starter for this kind of project. All we will be 
able to explain are various aspects of the feeling or sense of agency. Yet as we saw above, there can be a wide disconnect between feelings of agency and genuine agency. If what we are interested in is the neurophysiological basis of feelings of agency, then this is the right place to start. But we should take great care not to conflate the phenomenology of agency, in the sense of feelings of acting or willing, with actual agency. An explanatory project looking to expand the neurophysiological explanations of time consciousness to include the relationships between time and agency will need a different starting point than Husserlian phenomenology.

\section{Explaining temporal representation in agency}

This section takes up options for developing a parallel project to that of explaining the tripartite structure of time consciousness, a project capable of grounding the intentional content of temporal representations involved in agency in neurophysiological processes. Husserl's account was useful as a description of the explanandum, to which we then turn to neuroscience for an explanation. What would a candidate explanandum look like for a project involving agency as well? In a recent book, Michael Thompson (2008) offers an account of what he calls naïve action theory which, in my view, could provide just what we have been looking for. First, we'll examine some key elements of his views on naïve action theory and its implications for the representation of time in agency. Then, I'll motivate the connection between naïve action theory and Husserl's phenomenology, and finally, sketch out how a project could connect features of Thompson's account to neurophysiology in a manner analogous to that of neurophenomenology.

Naïve action theory is the practice of answering questions about what we are doing by providing another action as a rationalizing answer. ${ }^{v}$ It is explaining one action with another action. When asked why I am sifting through a pile of papers, I might explain this action of paper-sifting by citing another action, such as organizing my desk. I perform the one action - organizing my desk - in part by performing another - sifting through this pile of papers. This is in contrast to traditional accounts of rationalizing explanation, where it is assumed that action must be explained by something that is not itself an action: it might be a belief-plus-desire pair, or a motivating belief, a reason, a trying, or some other related notion (Anscombe 1969; Davidson 1963; Setiya 2003; Hornsby 1980; McDowell 1982). The rationalization of one action by another action has a particularly interesting form, since the action that does the work of rationalizing subsumes the rationalized action as a stage or component. The baking of a loaf of bread is an action that might explain, or rationalize, my taking the flour off the shelf right now. If someone asks what I am doing as I reach for the flour, it would be explanatory to say of my reaching, "I am baking bread, and require flour to knead it." Likewise, organizing my desk is a temporally extended action that involves a number of distinct stages. It subsumes as a single stage the sifting through of this pile of papers in front of me; there may be 
stages that are already complete before I begin to sort that particular pile of papers, as well as others that have not yet commenced as I sort that pile of papers.

Traditionally, action theorists have drawn a divide that groups together the categories of wanting, trying, and intending to do X as relevantly similar, and distinguishes them from doing X. Actually doing something is opposed to the situation where nothing has yet been done but in which the agents wants, intends, or tries to do something. One of Thompson's goals it to redraw this divide, such that wanting to do $\mathrm{X}$, intending to do $\mathrm{X}$, trying to do $\mathrm{X}$, and doing $\mathrm{X}$ all fall together as four related forms of rationalization. These four forms differ in the strength of what they attribute to the "progress of the deed". Intending to do X attributes less progress to $\mathrm{X}$ than does trying to do $\mathrm{X}$; trying to do $\mathrm{X}$ attributes less progress to $\mathrm{X}$ than does doing $X$. Thompson portrays these relationships in a table of rationalizations, arranged so that spatial relations among the attributions codified in it also represent inferential relations: "I'm doing X because I am doing Y" implies, but is not implied by, "I am trying to do X because I am doing Y," which in turn implies but is not implied by intending and wanting as progressively weaker implications. These four forms - wanting, intending, trying, and doing - are then contrasted with actions that are done or completed, actions with no remaining incomplete stages.

For our purposes, the key part of Thompson's account comes when he connects the four different forms of action rationalization in terms of what unifies them as forming a coherent structure in opposition to actions that are done or complete. The four forms of rationalization are related in that they each represent the act to which they apply as unfinished yet in some stage of being in progress. This is in contrast to having done $\mathrm{X}$, which represents the act in question as completed and no longer in progress. The form of attribution itself thereby carries temporal information about the action in question, and portrays that action both as a temporally extended process and as having progressed to a certain stage of that process.

The four potentially rationalizing judgments differ in important ways, but formally their relation to completed action is in each case the same: what is represented as coiled up or incomplete or partial in them, is represented as unfurled or finished or whole in the other. Twisting Brentano's vocabulary, we can say that "try", "intend", and "want" express modes of "imperfective inexistence" (of an event- or process-form)-but modes of imperfective inexistence that, unlike that expressed by the simple progressive, find application only in connection with rational life and its like. (Thompson 2008, 131)

It was because Husserl's project was phenomenological that it was capable of providing a description of an explanandum that could then be explained by resources in neuroscience. Thompson's project is presented in terms of an analysis of linguistic structures relating to action and tense. How can it serve as a description of an explanandum that could be explainable by neuroscience? Thompson's invocation here of Brentano's notion of intentional inexistence provides the bridge 
we need to understand how his account could serve in a manner analogous to Husserl's. Both Husserl and Thompson explicate the self-contained intentional temporal content of certain representations, as a result of which both offer potential explananda for neuroscientific explanations.

The idea of intentional inexistence was utilized by Brentano (1995) as a means of characterizing the distinction between physical and mental phenomena. The term is sometimes mistakenly taken to imply that the objects of experience are nonexistent. Instead, intentional inexistence characterizes mental phenomena as about something that is given in its entirety within the experience itself. Intentionality, recall, is the aboutness of experience, and inexistence means that the objects of experience exist or are given in their entirety in those very experiences. Inexistence can be thought of as "in-existing". For Brentano, the intentional inexistence of mental phenomena was their primary characterizing mark: mental phenomena are both about something, yet also contain entirely within themselves that which they are about. For Brentano, inexistence entails immanence: that which the experience is about does not 'point outside' the experience itself, to something in the world.

Husserl took this idea from Brentano and applied it to the content of the absolute time-constituting flow of consciousness. The intentional object of an experience is fully (not perspectivally) given in the very experience itself. This in-existing of that which experience is about, in the very experience itself, gives the absolute timeconstituting flow the characteristics it needs to render it possible for coherent sequences of events to be experienced, and for higher levels to be constituted out of the flow. It is also what makes the phenomenological bracketing of the external world possible: we can investigate the structure and content of temporal experience 'from within', as it were, and set aside the questions about what brings these structures of experience about, whether it accurately reflects the way things really are, etc. It is this feature of intentional inexistence that allows Husserl's account of time consciousness to serve as the explanandum for neuroscience.

This brings us back to Thompson. When we respond to a question about what we are doing with a naïve rationalization, by citing another action that we are also wanting, trying, intending, or doing (but have not yet done), we represent the action thereby explained as a temporal stage in an ongoing process that is partially, but only partially, completed. We represent both the content and the structure of what we are doing in temporal terms: we are engaged in doing $X$, and we are engaged in doing $\mathrm{Y}$, both of which will involve temporal details about the content of the actions themselves. And we temporally structure the representations of those actions themselves: to be doing $\mathrm{X}$ because we are doing $\mathrm{Y}$ is to represent $\mathrm{Y}$ as an ongoing, partially unfurled process, and to represent $X$ as standing in a very particular relation to $\mathrm{Y}$ by being one stage in the ongoing development of the unifying process of doing Y.

Thus, to attribute or claim agency in naïve action theory is to do so with a specific temporal inflection, to represent actions as standing in some temporal relationship 
to the present moment and to one another. "I did X" involves, in its very representational structure, the fact that the entire act $\mathrm{X}$ is completed, with no remaining stages. This attribution could be empirically inaccurate: I thought I did X, but it turns out something unseen prevented $\mathrm{X}$ from transpiring. Nevertheless, to say "I did X" is to represent oneself in a particular temporal position with respect to the action X. To say, "I am doing Z" is to represent oneself as partway through a temporally extended process that is the doing of $\mathrm{Z}$, where $\mathrm{Z}$ is represented as already begun but not yet complete. To say "I intend to do W" is, likewise, to represent $\mathrm{W}$ as a temporal process that one has not yet begun. We can consider the temporal representations involved in attributions of having done, doing, intending to do, and trying to do, without having to assume that these attributions are empirically accurate: we may not have done, nor be doing, what we think we have done or are doing; we may never begin what we currently intend to begin. Thus, we can bracket the question of empirical accuracy here and focus on the way in which the action is temporally represented.

It is these structural features of the representation of agency that make Thompson's account of naïve action theory a candidate for a project analogous to neurophenomenology. Doing something, being an agent, involves representations about how one's various actions, beliefs, intentions, and desires fit together which are fundamentally temporal in character, and treats agency as temporally extended and layered, with multiple time scales overlapping and nesting. When one does $\mathrm{X}$ because one is doing $\mathrm{Y}$, the representations involved in doing $\mathrm{X}$ must involve connections or references to doing $Y$, since that is the guiding and unifying action under which one is doing $\mathrm{X}$. And it must represent those actions as having specific temporal features that change through time, as the deed gets done, as Thompson puts it. The way in which one action is represented as a temporal stage in an encompassing, rationalizing, action is rich with possibilities for neuroscientific explanation.

What might an explanation of these features of temporal representation in agency look like? Developing the details of such an account lies beyond the purview of this paper, but a few suggestive remarks may serve. Consider the earlier discussion of action plans, which can be localized somewhat precisely in the frontal and prefrontal cortices, and which appear to serve as the guiding plans by which we implement specific motor sequences and evaluate and guide execution of movements through to conclusion. As we saw, there is evidence that action plans are formulated in terms of intended goals or expected outcomes, rather than in terms of the precise movements required to bring about those outcomes. Thompson's table of rationalizations would predict certain representational relationships between different action plans, and between updates to those action plans when actions are completed, especially shorter-term or more concrete actions that are stages in overarching or subsuming actions. It would be worthwhile to investigate how different action plans relate when one of the actions (like reaching for flour) is taken by the subject to be a stage in a further action plan (like baking bread). If Thompson's account is confirmed, premotor or motor processes should 
involve a representation of these relationships between nested action plans, and these action plans should change over time in ways compatible with naïve action theory.

Similarly, consider Thompson's divide between actions done on the one hand, and doing, trying, intending, and wanting on the other hand. If an agent takes herself to have completed an action, there should be relevant changes in the representational status of that action vis-à-vis these areas of the brain. Thompson's account can be taken as predicting major representational shifts for actions that have been completed, versus actions that are represented as pending or as in progress. There are many potential points of contact between Thompson's account and potential neurophysiological vehicles. These can be exploited in a two-way connection: Thompson's account may predict certain representational features to be explored that we might not have otherwise looked for; his account can be modified or disconfirmed in light of neurophysiological evidence.

\section{Conclusion}

Given the centrality to philosophy of questions concerning agency and temporal experience, there is a rather surprising lack of work that addresses the intersection of these topics. There is rich territory for exploration in the connections between time, agency, and representation. In this paper, I have briefly examined the way in which time and the representation of time figures in some major areas of philosophy.

When considering the issue of time and agency in psychology and the cognitive sciences, we saw that the representation of time, particularly the representation of temporal relationships between events, is affected by the exertion of agency. It also became apparent that time can be involved in agency in a myriad of ways without being explicitly represented; time may be involved in representational vehicles in the brain without being explicitly represented in experience. Sometimes the exertion of agency requires sophisticated representations of temporal processes. Here we saw that mismatches between expected and actual movements, including temporal gaps, can reduce the sense of agency, and that the sense of agency is not an adequate substitute for agency itself. We can then press the question about the ways in which time is not merely involved but also represented in experience.

Our examination of Husserl's account of time consciousness made clear that his account could not be generalized to include a substantive notion of agency, a weakness inherited by explanatory projects like neurophenomenology. Thompson's account of naïve action theory provided an alternative route to accommodate agency as well as temporal representation. I briefly sketched a project investigating the neural underpinnings of naïve action theory analogous to the projects in neurophenomenology. Thus, I hope to have laid out a number of intriguing avenues 
for future exploration with respect to the connections between time, agency, and representation. vi $^{\mathrm{i}}$

\section{References}

Brandom, Robert B. 1994. Making It Explicit: Reasoning, Representing, and Discursive Commitment. Cambridge, MA: Harvard University Press.

Bratman, Michael. 2007. Structures of Agency: Essays. Oxford University Press.

Brentano, Franz. 1995. Psychology from an Empirical Standpoint. Translated by Antos C. Rancurello, D.B. Terrell, and Linda L. McAlister. London, Routledge.

Burge, Tyler. 2010. Origins of Objectivity. Oxford University Press.

David, N., A. Newen, and K. Vogeley. 2008. "The 'Sense of Agency' and its Underlying Cognitive and Neural Mechanisms." Consciousness and Cognition 17(2): 523-534.

Dennett, Daniel C. and Marcel Kinsbourne. 1992. "Time and the Observer: The Where and When of Consciousness in the Brain." Behavioral and Brain Sciences 15: 183-201.

Ebert, J.P., and D.M. Wegner. 2010. "Time warp: Authorship Shapes the Perceived Time of Actions and Events." Consciousness and Cognition 19(1): 481-9.

Fagioli, S., B. Hommel, and R.I. Schubotz. 2007. "Intentional Control of Attention: Action Planning Primes Action-Related Stimulus Dimensions." Psychological Research 71(1): 22-29.

Flaherty, Michael G. 2011. The Textures of Time: Agency and Temporal Experience. Philadelphia, PA: Temple University Press.

Gallagher, Shaun. 2005. How the Body Shapes the Mind. Oxford University Press.

Gallagher, Shaun, and Dan Zahavi. 2008. The Phenomenological Mind: An Introduction to Philosophy of Mind and Cognitive Science. New York: Routledge.

Grush, Rick. 2004. "The Emulation Theory of Representation: Motor Control, Imagery, and Perception." Behavioral and Brain Sciences 27: 377-442.

Grush, Rick. 2006. "How to, and how not to, Bridge Computational Cognitive Neuroscience and Husserlian Phenomenology of Time Consciousness." Synthese. 153(3): 417-450. 
Haggard, Patrick, and Jonathan Cole. 2006. "Intention, Attention, and the Temporal Experience of Action." Consciousness and Cognition 16(2): 211-220.

Hinchman, Edward. 2003. "Trust and Diachronic Agency" Nous 37(1): 25-51.

Hommel, Bernhard. 2003. "Planning and Representing Intentional Action." The Scientific World Journal 3: 593-608.

Hoy, David Couzens. 2009. The Time of Our Lives: A Critical History of Temporality. Cambridge, MA: The MIT Press.

Husserl, Edmund. 2008. On the Phenomenology of the Consciousness of Internal Time (1893-1917). Translated by John Barnett Brough. Dordrecht: Kluwer Academic Publishers.

Kandel, Eric, James Schwartz, and Thomas Jessell. 2000. Principles of Neural Science. $4^{\text {th }}$ edition. New York: McGraw Hill.

Korsgaard, Christine. 2009. Self-constitution: Agency, Identity, and Integrity. Oxford University Press.

le Poivedin, Robin. 2011. “The Temporal Prison.” Analysis 71(3): 456-465.

Lloyd, Dan. 2002. "Functional MRI and the Study of Human Consciousness." Journal of Cognitive Neuroscience 14(6): 818-831.

Lutz, Antoine and Evan Thompson. 2003. "Neurophenomenology: Integrating Subjective Experience and Brain Dynamics in the Neuroscience of Consciousness." Journal of Consciousness Studies, 10(9-10): 31-52.

Moore, James, and Patrick Haggard. 2010. "Intentional Binding and Higher Order Agency Experience." Consciousness and Cognition 19(1): 490-491.

Preston, Catherine, and Roger Newport. 2010. "Self-Denial and the Role of Intentions in the Attribution of Agency." Consciousness and Cognition 19(4): 986-98.

Thompson, Michael. 2008. Life and Action: Elementary Structures of Practice and Practical Thought. Cambridge, MA: Harvard University Press.

van Gelder, Timothy. 1999. "Wooden Iron? Husserlian Phenomenology meets Cognitive Science." In Naturalizing Phenomenology: Issues in Contemporary Phenomenology and Cognitive Science, edited by Jean Petitot, Francisco J. Varela, Barnard Pacoud and Jean-Michel Roy, 245-265. Palo Alto, CA: Stanford University Press. 
Varela, Francisco J. 1996. "Neurophenomenology: A Methodological Remedy for the Hard Problem." Journal of Consciousness Studies 3(4): 330-349.

Zahavi, Dan. 2012. "The Time of the Self." Grazer Philosophische Studien (details pending).

Zahavi, Dan. 2003. Husserl's Phenomenology. Palo Alto, CA: Stanford University Press.

i By representation, I will here mean explicit representation in the contents of conscious experience. There are, of course, a range of other philosophically interesting issues related to other forms of representations of time (see Le Poivedin 2007).

ii There are also metaphysical questions about time and agency, such as are raised in Le Poivedin 2011. While interesting, these questions fall outside the purview of this paper.

iii I confine myself here to Husserl's work because his is the account on which most if not all attention has been focused for the neurophysiological explanatory project to be shortly discussed. Arguably, there is much richer material on time, agency, and representation in the so-called "continental' tradition. For an excellent account of temporality in the tradition following after Husserl, see Hoy 2009.

iv There are a few works in this arena, such as (Gallagher 2005), which do not focus on Husserl but include other phenomenologists as well. As such, this work is less susceptible to the problem outlined here with respect to agency.

$\checkmark$ To rationalize an action is, in a perhaps oversimplified form, to provide an explanation of actions in terms of reasons for acting, rather than a mere cause of one's movement.

vi Much thanks to Endre Begby for discussion and feedback on this work. Thanks also to participants in the SFU Explanation Seminar for their helpful questions on agency and explanation. 